\title{
The Autorepressor: a Case Study of the Importance of Model Selection
}

\author{
Andreas W. K. Harris ${ }^{\dagger}$, Ciarán L. Kelly ${ }^{\star}$, Harrison Steel ${ }^{\dagger}$, Antonis Papachristodoulou ${ }^{\dagger}$
}

\begin{abstract}
Major challenges exist in the design of gene regulatory networks. Some of these can be addressed by the in silico modelling and design of systems prior to implementation. However, reliable modelling of a given system is predicated upon a range of simplifying assumptions which may only be valid for a limited range of architectures and experimental conditions. In this paper we study the autorepressor, also referred to as the negative autoregulator, a genetic motif common both in natural and synthetic circuits. A number of approaches to modelling the autorepressor are presented, and one of these is extended to include the impact of inducer consumption, a phenomenon frequently observed in experiments. We implement this system using the tet-repressor (TetR), and compare the in vivo data with the results of simulations using parameters taken from the literature. We demonstrate that a modelling approach that considers inducer sequestration due its binding with a transcription factor may be required to qualitatively replicate experimental results. We conclude by drawing comparisons between experimental and simulated results, and discuss approaches by which modelling could be extended to better represent observed behaviours.
\end{abstract}

\section{INTRODUCTION}

Building reliable Synthetic Biological systems is challenging [1] due to factors including the inherent fluctuations and noise of the cellular environment [2], as well as unforeseen interactions between biological components and systems [3]. To address these challenges Synthetic Biologists have attempted to introduce elements from control engineering [4], [5], [6], inspired by a range of natural biological systems known to utilise analogous architectures [7]. The design of such control architectures is informed by extensive in silico modelling, which has motivated the development of a range of mathematical approaches describing biological networks and systems [8]. However, these mathematical frameworks are built upon a broad array of assumptions which may in some cases be violated, thereby undermining the performance of systems they have been used to design [9].

The autorepressor (or negative autoregulator) is a common genetic control motif, consisting of a gene that encodes a protein repressing its own expression (Fig. 1a). This architecture has been well-studied in various contexts [10], [11], [12], [13], and is employed ubiquitously to control expression of repressors in both prokaryotes [14] and eukaryotes [15]. Since it represents a standard, minimal, regulatory unit, the

$\dagger$ : Authors with the Department of Engineering Science, University of Oxford, Oxford, OX1 3PJ, UK. e-mail: (\{andreas.harris, harrison.steel, antonis\}@eng.ox.ac.uk). $\star$ : Author with the Centre for Synthetic Biology and Innovation, Imperial College London, London, SW7 2AZ, UK. email: ciaran.kelly@imperial.ac.uk. A.W.K Harris is supported by the Engineering and Physical Sciences Research Council (EPSRC) Doctoral Training Centre in Systems Biology, University of Oxford. H. Steel is supported by the General Sir John Monash Foundation. A. Papachristodoulou is supported in part by EPSRC projects EP/J012041/1 and EP/M002454/1. autorepressor can be used as a model system to compare in silico and in vivo results for gene regulatory networks. Models of the autorepressor vary in complexity, from singlestate descriptions to more complex reaction networks that include both transcription and translation processes [10], most of which rely on the Hill function to describe the mechanism of repression. The selection of a particular model is informed by the need to capture sufficient richness of behaviour for a given design task, whilst simultaneously minimising the number of model parameters that must be selected. This is critical for the design of many systems in which desirable behaviours (for example, oscillations) may only emerge for limited ranges of parameter values [16], [17]. Thus, valid correspondence between in silico and experimental results is predicated upon reliable selection of a modelling framework and parameter values.

In this paper we have constructed a biological implementation of the autorepressor, using the repressor (TetR) and promoter $\left(\mathrm{P}_{t e t}\right)$ of the tetracycline (tc)-resistance gene cluster found in many bacteria (Fig. 1b), similar to circuits in [11], [13]. In Section II we describe the biological implementation of our autorepressor. In Section III we then present a biochemical description of its behaviour, and identify appropriate parameter values from the literature. In Section IV we simulate dose responses for 1- and 3-state models of the autorepressor, and compare these results to experimental data. Based on these comparisons, in Section V we extend the 3-state model to create a 4-state model that accounts for consumption of inducer molecules, and compare this with additional data. Finally, Section VI concludes the paper.

\section{BIOLOGICAL IMPLEMENTATION}

To benchmark different modelling approaches, we constructed a biological implementation of autorepression using TetR, the repressor of the tetracycline (tc)-resistence gene cluster (Fig. 1b). Two recently made autorepressor plasmids pCK200 and pCK210 (as pCK200 but with lacI replaced with another gene not used in this study; no fluorescence/growth differences between either plasmid) were used in this biological implementation of the autorepressor circuit [18]. These plasmids consist of $a m p R$ (confers ampicillin resistance), the pBR322 origin of replication (resulting in a copy number of $15-20$ per cell [19]), the tet promoter $\left(\mathrm{P}_{t e t}\right)$, a synthetic RBS (with a translation initiation rate of 50,000), and a tetR-sfGFP fusion gene. The fusion of the TetR protein to sfGFP allows real-time quantification of protein production (and thus gene expression). TetR, in the absence of the inducer (anhydrotetracycline, aTc), is free to bind to the DNA recognition sites in $\mathrm{P}_{\text {tet }}$. This prevents 


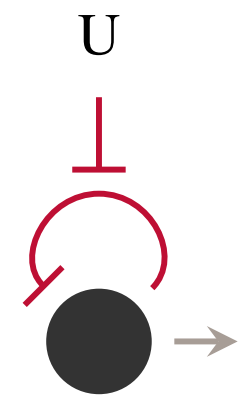

(a) Minimal Representation

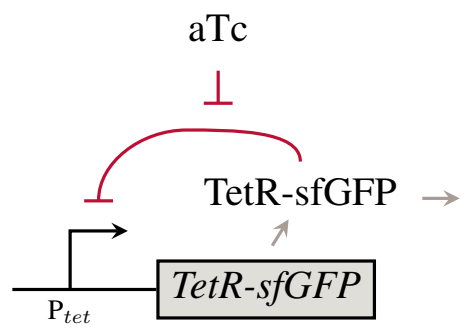

(b) Biological Circuit
Fig. 1: (a) The minimal representation of the autorepressor. The black dot represents a transcription factor (TF), the blunt arrows represent repression, and the pointed arrow represents a system output. The inducer, $\mathrm{U}$, inhibits the expression of the TF. (b) The biological parts used in the circuit studied in this paper.

RNA polymerase from binding to the DNA, thus halting expression from this promoter. TetR binds to free aTc (when it is present) with high affinity, resulting in a conformational change in TetR which prevents its binding to $\mathrm{P}_{t e t}$, thus allowing transcription and subsequently translation to occur. A typical working concentration of the inducer aTc is 0

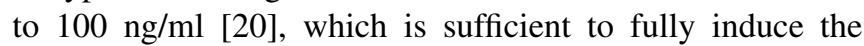
promoter, whilst not excessively inhibiting cell growth.

The wild-type laboratory E. coli strain, MG1655 was used for all experiments. Cells were transformed with the autorepressor plasmid (pCK200 or pCK210) and transformants used to inoculate $\mathrm{EZ}$ rich defined media (Teknova Inc), which were grown overnight at $37^{\circ} \mathrm{C}$ with vigorous shaking. These starter cultures were diluted to an optical density $\left(\mathrm{OD}_{600 \mathrm{~nm}}\right)$ of 0.05 in $300 \mu \mathrm{l}$ of fresh EZ rich defined media and grown in deep well multiplates at $37^{\circ} \mathrm{C}$ with rapid shaking. At specified time points, samples were taken and diluted 1:10 into phosphate-buffered saline (PBS) to a total volume of 200 $\mu 1$. These samples were then loaded into the autosampler of an Attune NxT Flow Cytometer (ThermoFisher Scientific) and GFP fluorescence was measured. Data shown represent the geometric mean of 10,000 events (cells).

In plate-reader experiments, transformants were grown in $200 \mu \mathrm{l}$ of EZ rich defined media in shallow 96-well plates and incubated at $37^{\circ} \mathrm{C}$ with vigorous shaking in a FLUOstar Omega microplate reader (BGM Labtech), which measured $\mathrm{OD}_{600 \mathrm{~nm}}$ and GFP fluorescence at regular intervals.

\section{BiochemiCAl Model of the Autorepressor}

We begin by building a biochemical reaction network that describes the autorepressor, taking specific details of the tet-repressor mechanism into account. Since TetR dimerises with a very high affinity [21] we neglect to model this process, assuming TetR only exists in dimer form (the form in which it is active). Furthermore, we assume that the free TetR dimer is the only species that can interact with the $\mathrm{P}_{\text {tet }}$ operator region, which is justified since the binding of aTc to TetR reduces TetR's DNA binding affinity by many orders of magnitude [14]. Thus, the TetR autorepressor can be represented by a chemical reaction network of the form:

$$
\begin{aligned}
& \mathrm{M} \stackrel{\alpha_{P}}{\longrightarrow} \mathrm{M}+\mathrm{P}, \quad \mathrm{P} \stackrel{\delta_{P}}{\longrightarrow} \emptyset, \\
& \mathrm{G} \stackrel{\alpha_{M}}{\longrightarrow} \mathrm{G}+\mathrm{M}, \quad P_{U} \stackrel{\delta_{P}}{\longrightarrow} \emptyset, \\
& \mathrm{P}+\mathrm{U} \underset{k_{-1}}{\stackrel{k_{1}}{\rightleftarrows}} P_{U}, \quad P_{G} \stackrel{\delta_{P}}{\longrightarrow} \mathrm{G}, \\
& \mathrm{P}+\mathrm{G} \underset{k_{-2}}{\stackrel{k_{2}}{\rightleftharpoons}} P_{G}, \quad \mathrm{M} \stackrel{\delta_{M}}{\longrightarrow} \emptyset,
\end{aligned}
$$

where $\mathrm{P}$ is the concentration of free TetR dimer protein, $\mathrm{M}$ is the concentration of mRNA encoding the TetR-sfGFP fusion protein, $\mathrm{G}$ is the free gene concentration, and $\mathrm{U}$ is the inducer concentration. The complexes $P_{U}$ and $P_{G}$ are the TetR-inducer and the TetR-gene complexes respectively. The rate of transcription is $\alpha_{P}$ and the rate of translation is $\alpha_{M}$. We assume the degradation rate of TetR $\left(\delta_{P}\right)$ is unaffected by its bound state, and that mRNA degrades with rate $\delta_{M}$. We model the binding of TetR and inducer or gene as reversible reactions with forward/reverse rate constants of $k_{ \pm 1}$ and $k_{ \pm 2}$ respectively. Gene concentration is also conserved, with $G_{t o t}=G+P_{G}$, where $G_{t o t}$ is total gene copy number, which in this case is equal to the plasmid copy number.

For the biological implementation of this system the experimental output is measured fluorescence intensity. This is used as a proxy for the concentration of TetR, since each TetR protein is tagged with the fluorescent protein sfGFP. Thus, the measured output $(\mathrm{Y})$ is the combination of all species that include TetR:

$$
Y=P+P_{U}+P_{G} .
$$

For simulations we used parameter values (presented in Table I) from the literature as follows. The doubling time of the cell is assumed to be $28 \mathrm{~min}$, which yields $\delta_{P}$. The turn over rate of mRNA is assumed to be 10 times this [22], giving $\delta_{M}$. We set the transcription rate $\left(\alpha_{M}\right)$ to a reasonable biological value of $1 \mathrm{~min}^{-1}$, and set the translation rate $\left(\alpha_{P}\right)$ to half this value since TetR is assumed to quickly dimerise to create its functional form. We generally have $K_{I}<K_{G}$ [23] because the interaction between TetR and aTc is stronger than that between TetR and its operator region. We select values from systems similar to ours, resulting in $K_{G}=10^{-11} \mathrm{M}$ [24] and $K_{I}=10^{-12} \mathrm{M}$ [23]. The value of $k_{1}$ is from [25] and the value of $k_{-2}$ is calculated from the half-life of the TetR-operator complex from [26]. The gene concentration $G_{t o t}$ is consistent with the pBR322 oriregion used on the plasmid, which has a copy number of about 17 [19], in a cell of volume $10^{-15} 1$. We convert to the inducer units of $\mathrm{ng} \mathrm{ml}^{-1}$ in simulations using a molecular weight for aTc of $426 \mathrm{~g} \mathrm{~mol}^{-1}$.

\section{Steady State Dose Response}

In this section, we show the importance of model selection for predicting the dose response of the autorepressor. To describe the autorepressor we interpret the biochemical reactions detailed in (1) in two different ways, providing two different models. 


\section{A. Single State ODE Model}

The most common mathematical model describing the autorepressor is a single-state model, employing a Hill function to model the repression mechanism [10], [11], [12], [14]. Here we include the inducer concentration in the Hill function, so that it can be treated as an explicit input to the system. The single-state model is thus expressed as:

$$
\dot{Y}=\frac{\alpha G_{t o t} K_{G}\left(K_{I}+U\right)}{K_{G}\left(K_{I}+U\right)+K_{I} Y}-\delta_{P} Y,
$$

where $Y$ is the concentration of repressor protein (TetR) and $U$ is the concentration of inducer. Also, $\alpha=\frac{\alpha_{P} \alpha_{M}}{\delta_{M}}$ is the maximal expression rate, $G_{t o t}$ is the total concentration of gene, $\delta_{P}$ is the degradation rate of the repressor protein, and $K_{G}=\frac{k_{-2}}{k_{2}}$ and $K_{I}=\frac{k_{-1}}{k_{1}}$ are dissociation constants for the interactions between the repressor and gene, and the repressor and inducer respectively.

The on-off reactions between the repressor, and inducer and gene in (1) are reduced to rational functions using standard methods [8], [27], resulting in the Hill function. The Hill coefficient used is $n=1$, which is consistent with the literature [11], [14]. This process reduces the number of states required to model the system by two, which is further reduced to one by modelling transcription and translation in a single step (with rate $\alpha$ ), on account of the time scale separation between the life-times of mRNA and protein.

Setting $\dot{Y}=0$ the steady state TetR concentration $\left(Y^{s s}\right)$ is:

$$
Y^{s s}(U)=\frac{K_{G}\left(K_{I}+U\right)}{2 K_{I}}\left[\sqrt{1+\frac{4 \alpha G_{t o t} K_{I}}{\delta_{P} K_{G}\left(K_{I}+U\right)}}-1\right] .
$$

In the limit of large $U$ (inducer saturation) this expression simplifies to $Y^{s s} \approx \frac{\alpha G_{\text {tot }}}{\delta_{P}}$. This is the expression expected when there is no autorepression, or equivalently, if $K_{G}$ is large: The mechanistic equivalent of very weak binding between TetR and the $P_{\text {tet }}$ operator region.

\section{B. Three State ODE Model}

Instead of simplifying our system's dynamics via expression as a single Hill function, we can utilise a system of ODEs to model the TetR, inducer-TetR complex, and free gene concentrations separately:

$$
\begin{aligned}
\dot{P}_{U} & =k_{1} P U-\left(k_{-1}+\delta_{P}\right) P_{U}, \\
\dot{G} & =-k_{2} P G+\left(k_{-2}+\delta_{P}\right)\left(G_{t o t}-G\right), \\
\dot{P} & =\alpha G-\delta_{P} P-k_{1} P U+k_{-1} P_{U} \\
& -k_{2} P G+k_{-2}\left(G_{t o t}-G\right) .
\end{aligned}
$$

This system of equations more accurately reflects the reactions included in (1), though it again assumes that expression can be modelled as a single step with rate $\alpha$. The system's output, which corresponds to the fluorescence of the TetRsfGFP fusion proteins and is noted in (2), can be expressed in terms of the variables used in (5) as:

$$
Y=P+P_{U}+G_{t o t}-G \text {. }
$$

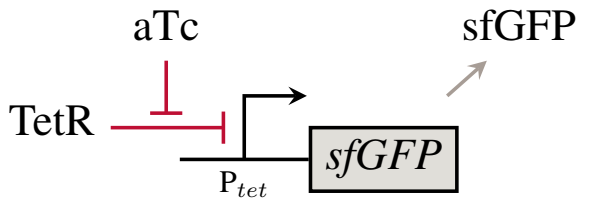

Fig. 2: Biological circuit topology of open-loop expression repressed by TetR through the $\mathrm{P}_{\text {tet }}$ promoter, similar to that in [20].

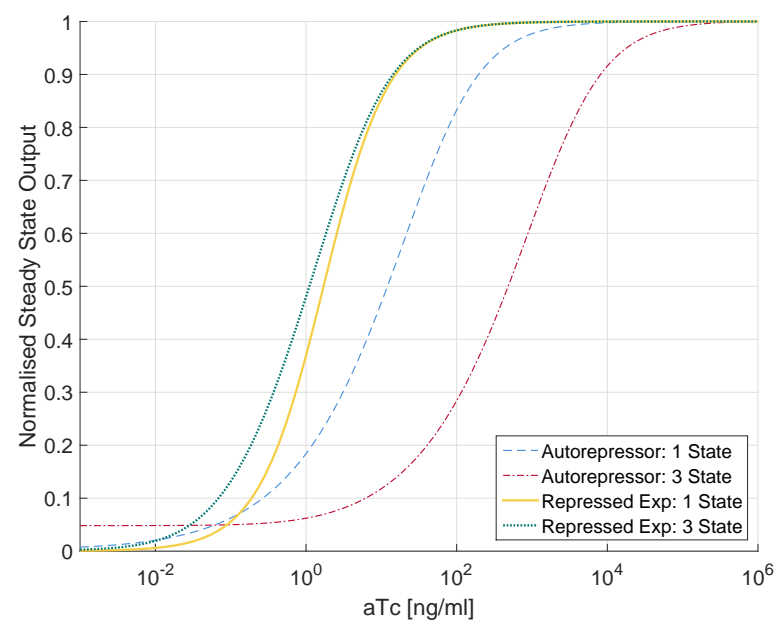

Fig. 3: Simulated dose responses of the single- and threestate models of the autorepressor (Eqns. (3) and (5) respectively) and repressed expression (Eqns. (7) and (8)).

\section{For Comparison: Repressed Expression}

The open-loop TetR repression mechanism, in which TetR is constitutively expressed (Fig. 2), is frequently used for control in synthetic circuits, and has a dose response that is well-known [20], [24]. By way of comparison to (3), based on a chemical reaction network very similar to (1), a singlestate ODE model of repressed expression of a gene using a Hill function can be derived:

$$
\dot{Y}=\frac{\alpha G_{t o t} K_{G}\left(K_{I}+U\right)}{K_{G}\left(K_{I}+U\right)+K_{I} P_{t o t}}-\delta_{\mathrm{P}} Y,
$$

where $Y$ in this case is the concentration of reporter protein (sfGFP in Fig. 2), $P_{t o t}$ is the bulk concentration of the repressor TetR, which is constitutively expressed in the cell, and other parameters are defined analogously to before.

As in the case of the autorepressor, a more detailed threestate model of this system can be constructed avoiding the Hill function:

$$
\begin{aligned}
\dot{Y} & =\alpha G-\delta_{\mathrm{P}} Y, \\
\dot{G} & =-k_{2} P G+k_{-2}\left(G_{t o t}-G\right), \\
\dot{P} & =-k_{1} P U+k_{-1}\left(P_{t o t}-P-G_{t o t}+G\right) \\
& -k_{2} P G+k_{-2}\left(G_{t o t}-G\right),
\end{aligned}
$$

where parameters are defined analogously to before. 
TABLE I: Parameters values used in simulation, taken/calculated from the literature as described in Section III. $P_{t o t}$ is selected so that the repressed expression simulations in Fig. 3 approximate those in [20].

\begin{tabular}{c|c|c|c} 
Param. & Value & Param. & Value \\
\hline$\alpha_{\mathrm{P}}$ & $0.5 \mathrm{~min}^{-1}$ & $k_{1}$ & $0.42 \mathrm{nM}^{-1} \mathrm{~min}^{-1}$ \\
$\delta_{\mathrm{P}}$ & $0.025 \mathrm{~min}^{-1}$ & $k_{-1}$ & $4.2 \times 10^{-4} \mathrm{~min}^{-1}$ \\
$\alpha_{\mathrm{M}}$ & $1 \mathrm{~min}^{-1}$ & $k_{2}$ & $5.8 \mathrm{nM}^{-1} \mathrm{~min}^{-1}$ \\
$\delta_{\mathrm{M}}$ & $0.25 \mathrm{~min}^{-1}$ & $k_{-2}$ & $5.8 \times 10^{-2} \mathrm{~min}^{-1}$ \\
$K_{I}$ & $10^{-3} \mathrm{nM}$ & $G_{\text {tot }}$ & $28.2 \mathrm{nM}$ \\
$K_{G}$ & $10^{-2} \mathrm{nM}$ & $P_{\text {tot }}$ & $40 \mathrm{nM}$
\end{tabular}

\section{Simulated Dose Response}

In Fig. 3 we simulate dose response curves for the models in (3), (5), (7), and (8) using the parameters presented in Table I. The open-loop repressed expression profiles correspond well with those in Fig. 4a of [20]; full saturation is achieved when the inducer concentration reaches approximately $25 \mathrm{ng}$ $\mathrm{ml}^{-1}$. The behaviour of the single- and three-state models of the open loop system correspond closely, demonstrating that the single state model of repressed expression is a singularlyperturbed reduction of the three state case.

However, this is not true for the simulated autorepressor, for which the dose response curves for the one- and threestate models show sensitivities to inducer concentration that differ substantially. This difference is caused by inaccuracies in the reduction in (3) which predominantly arise because we have $k_{-1} \ll \delta_{P}$. If we set $K_{I}=\delta_{P} / k_{1}$ in (3), then the single-state model for the autorepressor closely aligns to the outcome of the three-state model in Fig. 3.

\section{E. The in vivo Dose Response}

To assess the behaviours predicted by these models, the dose response of the autorepressor was then experimentally measured using the methods detailed in Section II. The geometric mean of 10,000 events in a flow cytometer was calculated to give the average fluorescence per cell in a population, which was averaged over biological triplicates, and is presented in Fig. 4. The dose response of the autorepressor (Fig. 4a) begins to rise at $[\mathrm{aTc}] \approx 10 \mathrm{ng} \mathrm{ml}^{-1}$, and is still not saturated at $[\mathrm{aTc}]=400 \mathrm{ng} \mathrm{ml}^{-1}$, as predicted by the three-state model (5) in Fig. 3. We do not observe a clear saturation in the measured dose response; for the higher inducer concentrations (1000 and $4000 \mathrm{ng} \mathrm{ml}^{-1}$ ) the output is significantly decreased. This is likely a result of burden on the cell due to the high concentrations of toxic aTc, which can slow or prevent growth and gene expression. Fig. 4b demonstrates that for $[\mathrm{aTc}]=1000 \mathrm{ng} \mathrm{ml}^{-1}$ the output is still rising at the end of the experiment, and thus may yield a higher output as predicted in Fig. 3. However, when inducer concentration is increased further $\left([\mathrm{aTc}]=4000 \mathrm{ng} \mathrm{ml}^{-1}\right.$ ) the high burden/toxicity results in a monotonic decrease in fluorescence throughout the experiment.

There are thus two primary qualitative differences between our experimental and simulated results. First is the impact of aTc toxicity, which leads to decreased output at high induction levels. This is an obvious direction for further
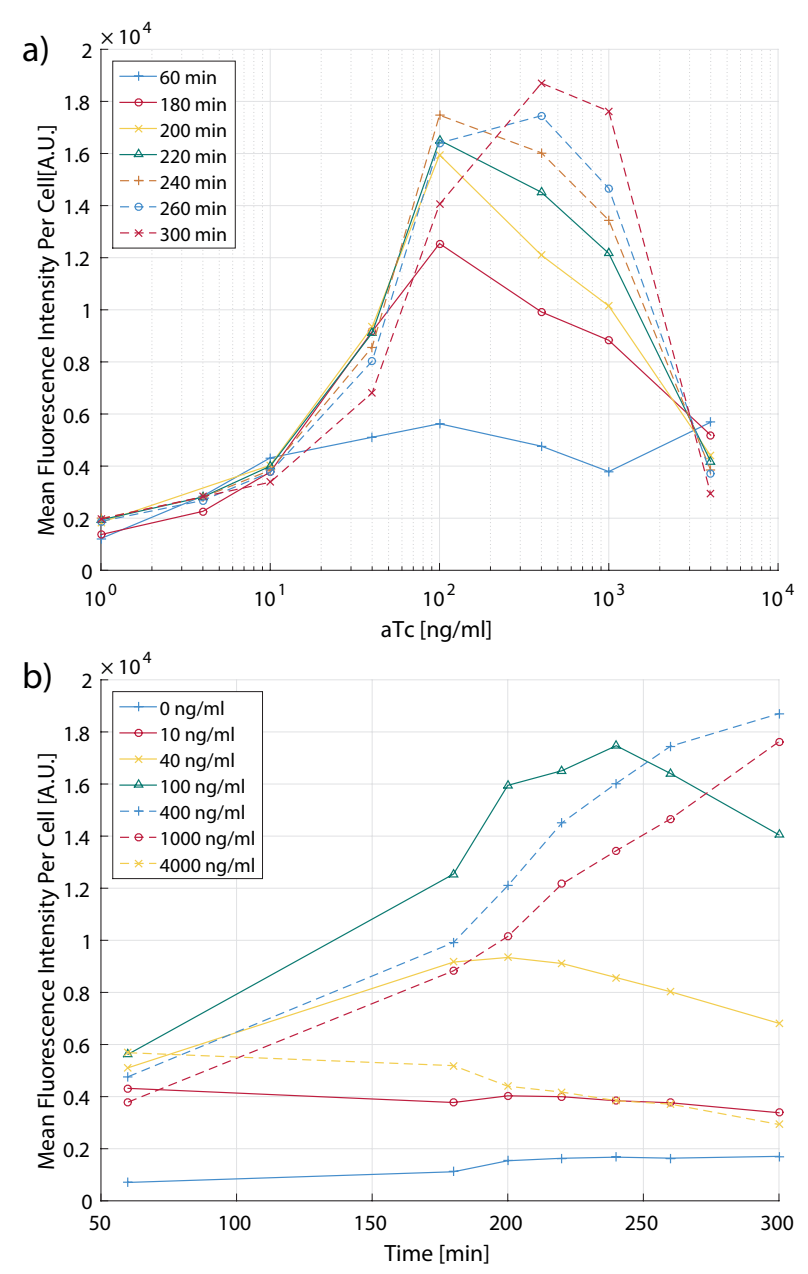

Fig. 4: Flow cytometry data from the circuit encoded by plasmid pCK210 illustrating (a) The dose response of TetR autorepressor fluorescence to increasing concentrations of anhydrotetracylcline (aTc). (b) Time course of TetR autorepressor fluorescence at various concentrations of aTc.

research. The second is that rather than reaching a steadystate, fluorescence output is observed to peak (earlier for lower inducer concentrations) and then decrease over time (clearly observable for $[\mathrm{aTc}]=40,100 \mathrm{ng} \mathrm{ml}^{-1}$ in Fig. 4b). We hypothesise that this is caused by sequestration of a limited pool of aTc, which we investigate in the next section.

\section{The Time Course Dynamics}

Motivated by the potential aTc sequestration observed in the previous section, we extend our three-state model to reflect this and analyse the system's time course behaviour.

\section{A. Extended Four State ODE Model}

Inducer ( $\mathrm{aTc}$ ) depletion due to its binding with TetR is known to occur experimentally [28], but is not included in common modelling frameworks [10], [11], [14]. In many systems, when the tet-repressor is in open loop, this factor is justifiably ignored because TetR concentrations are sufficiently low to leave most aTc unbound. However, the 
negative feedback in the closed-loop autorepressor means that when aTc is added to the system, transcription and therefore expression of the TetR-sfGFP fusion continues until virtually all of the aTc is sequestered. This results in a total concentration of the repressing fusion protein that is almost equivalent to that of the inducer. Dividing cells continue to sequester inducer (and share the expressed repressorinducer complex) until there is none left in solution, at which point repression kicks in, preventing further production of the fluorescing repressor. Subsequently the cells continue to divide, diluting the previously expressed repressor and causing the fluorescence per cell to drop. Expanding (5) to include this mechanism, our model takes the form:

$$
\begin{aligned}
\dot{P}_{U} & =k_{1} P U-\left(k_{-1}+\delta_{P}\right) P_{U}, \\
\dot{G} & =-k_{2} P G+\left(k_{-2}+\delta_{P}\right)\left(G_{t o t}-G\right), \\
\dot{U} & =-k_{1} P U+k_{-1} P_{U}, \\
\dot{P} & =\alpha G-\delta_{P} P-k_{1} P U+k_{-1} P_{U} \\
& -k_{2} P G+k_{-2}\left(G_{t o t}-G\right),
\end{aligned}
$$

where the new state $U$ records the concentration of the free inducer. The inducer can be in two forms: free $(U)$ and bound to repressor $\left(P_{U}\right)$. In the form where it is bound to repressor, it degrades, simulating the dilution of the protein as cells divide. This simple model treats the inducer as a finite resource that is consumed over time. In reality this process would be slowed since some aTc from the growth media would be transported into the cell over time.

A comparison of the three- (5) and four-state models (9) is presented in Fig. 5. The three-state model settles down to a non-zero steady state, whereas the four-state model settles at zero, peaking at higher values for higher levels of inducer. Additionally, the peak fluorescence values are predicted to occur at later times for higher concentrations of inducer.

\section{B. In vivo Time Course}

We now perform plate-reader experiments to more accurately characterise the time-course performance of our system, for which data is presented in Fig. 6. As predicted by the four-state model (9), the fluorescence can be seen to rise and then fall as the inducer aTc is sequestered in the cell and in effect 'consumed' (though part of this fall is due to increasing OD whilst minimal fluorescent reporter is expressed). The peaking of fluorescence values also occurs in a staggered manner, according to the initial concentrations of inducer, with higher concentrations peaking at later times. These qualitative traits parallel those in observed in Fig. 4b, and are in agreement with the simulations of the extended model (9) in Fig. 5. The optical density of the cell cultures (Fig. 6b) demonstrates a reduction in growth for increased aTc concentrations, though at these concentrations toxicity has only a minor effect.

\section{CONCLUSION}

We found that (compared to open-loop expression) an autorepressing (closed-loop) system is sensitive to higher inducer concentrations, which are sufficiently large to prevent

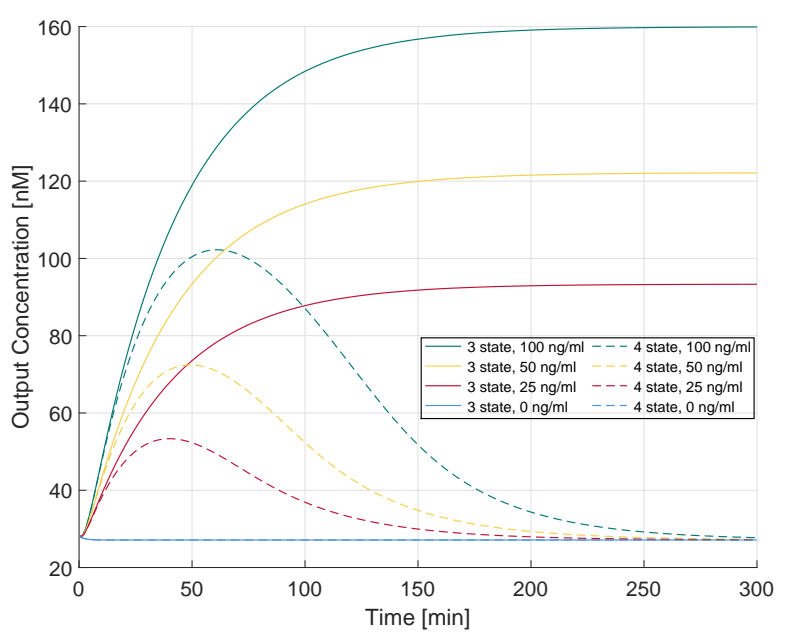

Fig. 5: Simulated time course data for the three- and fourstate models (Equations (5) and (9) respectively). As TetR is produced, for the four state model all aTc is eventually bound, after which any TetR produced is able to repress it's own expression (and hence that of GFP).

the system reaching a true steady-state dose-response due to the inducer's toxicity. We also find that, because TetR concentrations are higher in the closed-loop case, consideration of inducer sequestration is vital for accurate modelling of the in vivo behaviour of the autorepressor. Future work could include extending models to consider cell growth behaviour (as measured in Fig. 6b), as well as fitting models to data and utilising structural identifiability methods [29] to assess which parameters can be ascertained from a given data set. Furthermore, different experimental procedures that utilise microfluidics or a turbidostat setup to regulate inducer concentration could be employed to investigate sequestration mechanisms. Overall, this study highlights the importance of careful model selection when combining theory and experiment, particularly to include the impact of inducer sequestration when feedback architectures are utilised.

\section{REFERENCES}

[1] A. A. K. Nielsen, T. H. Segall-Shapiro, and C. A. Voigt, "Advances in genetic circuit design: Novel biochemistries, deep part mining, and precision gene expression," Current Opinion in Chemical Biology, no. 6, pp. 878-892, 2013.

[2] G. Balázsi, A. Van Oudenaarden, and J. J. Collins, "Cellular decision making and biological noise: From microbes to mammals," Cell, no. 6, pp. 910-925, 2011.

[3] D. Del Vecchio, "Modularity, context-dependence, and insulation in engineered biological circuits," Trends in Biotechnology, no. 2, pp. 111-119, 2015

[4] D. Del Vecchio, A. J. Dy, and Y. Qian, "Control theory meets synthetic biology." Journal of the Royal Society, Interface / the Royal Society, no. 120, 2016.

[5] T. P. Prescott and A. Papachristodoulou, "Layered decomposition for the model order reduction of timescale separated biochemical reaction networks." Journal of theoretical biology, pp. 113-22, sep 2014.

[6] A. Harris, J. Dolan, C. Kelly, J. Anderson, and A. Papachristodoulou, "Designing Genetic Feedback Controllers," IEEE Transactions on Biomedical Circuits and Systems, no. 4, pp. 475-484, 2015. 

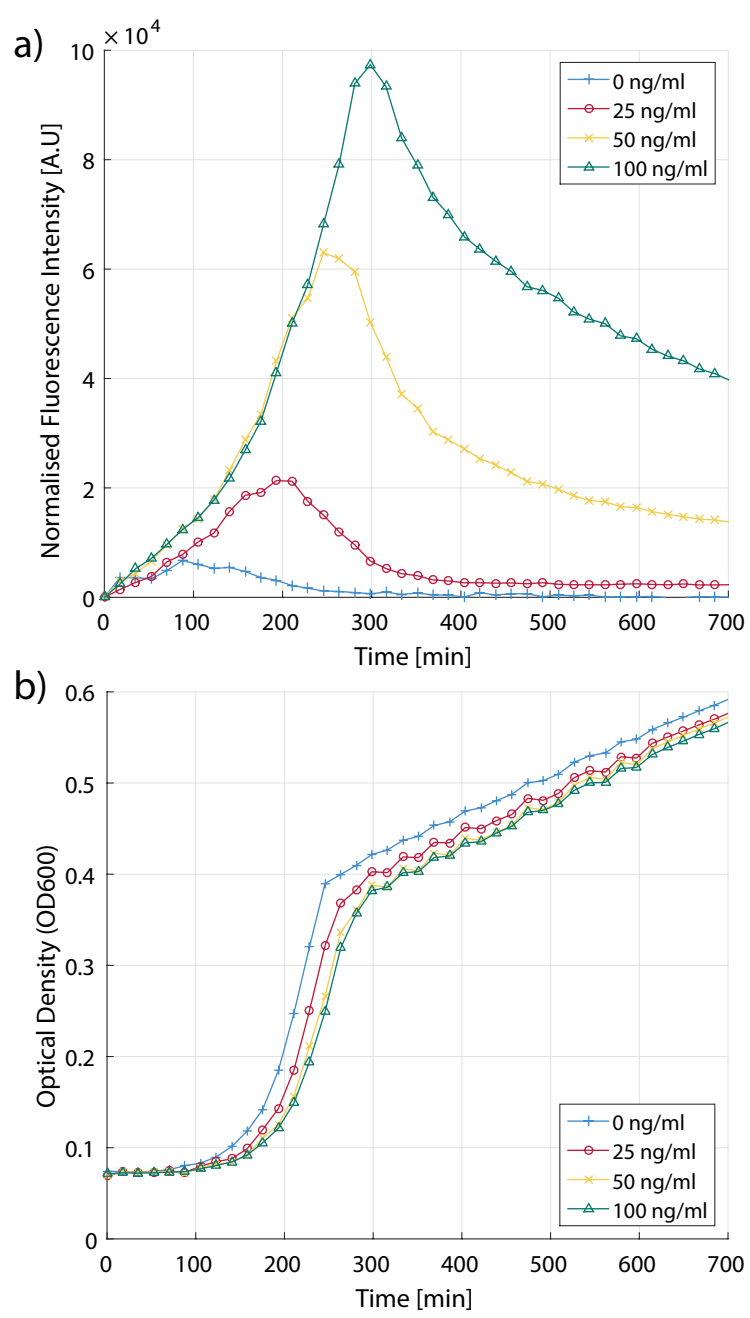

Fig. 6: Plate reader data from the circuit encoded by plasmid pCK200 illustrating (a) Fluorescence over time for the autorepressor system with different concentrations of aTc. Mean fluorescence values of three biological replicates normalised by optical density $\left(\mathrm{OD}_{600 \mathrm{~nm}}\right)$. (b) Growth $\left(\mathrm{OD}_{600 \mathrm{~nm}}\right)$ of MG1655 cells carrying the autorepressor plasmid corresponding to the data presented in (a).

[7] M. Khammash, "An engineering viewpoint on biological robustness." BMC biology, no. 1, p. 22, 2016.

[8] B. Ingalls, Mathematical Modelling in Systems Biology: An Introduction. Cambridge, Massachusetts: The MIT Press, 2013.

[9] E. Hancock, G. Stan, J. Arpino, and A. Papachristodoulou, "Simplified mechanistic models of gene regulation for analysis and design," Journal of the Royal Society, Interface, no. 108, pp. 1-33, 2015.

[10] U. Alon, "Network motifs: theory and experimental approaches." Nature Reviews. Genetics, no. 6, pp. 450-61, 2007.

[11] A. Becskei and L. Serrano, "Engineering stability in gene networks by autoregulation." Nature, no. June, pp. 590-593, 2000.

[12] D. Nevozhay, R. Adams, K. Murphy, K. Josic, and G. Balázsi, "Negative autoregulation linearizes the dose-response and suppresses the heterogeneity of gene expression." Proceedings of the National Academy of Sciences, no. 13, pp. 5123-8, 2009.

[13] D. Austin, M. Allen, J. McCollum, R. Dar, J. Wilgus, G. Sayler, N. Samatova, C. Cox, and M. Simpson, "Gene network shaping of inherent noise spectra." Nature, no. 7076, pp. 608-11, feb 2006.

[14] N. Rosenfeld, M. Elowitz, and U. Alon, "Negative autoregulation speeds the response times of transcription networks," Journal of Molecular Biology, no. 5, pp. 1-7, 2002.
[15] T. Lee, N. Hannett, C. Harbison, C. Thompson, I. Simon, J. Zeitlinger, E. Jennings, H. Murray, D. Gordon, B. Ren, J. Wyrick, J. Tagne, and R. Young, "Transcriptional Regulatory Networks in Saccharomyces cerevisiae," Science, pp. 799-804, 2002.

[16] M. Elowitz and S. Leibler, "A synthetic oscillatory network of transcriptional regulators." Nature, no. 6767, pp. 335-8, jan 2000.

[17] T. Shopera, W. R. Henson, A. Ng, Y. J. Lee, K. Ng, and T. S. Moon, "Robust, tunable genetic memory from protein sequestration combined with positive feedback," Nucleic Acids Research, no. 18, pp. 90869094, 2015.

[18] C. L. Kelly, A. W. K. Harris, H. Steel, E. J. Hancock, J. T. Heap, and A. Papachristodoulou, "Synthetic negative feedback circuits using engineered small rnas," bioRxiv, 2017.

[19] C. Lee, J. Kim, S. G. Shin, and S. Hwang, "Absolute and relative QPCR quantification of plasmid copy number in Escherichia coli," Journal of Biotechnology, no. 3, pp. 273-280, 2006.

[20] R. Lutz and H. Bujard, "Independent and tight regulation of transcriptional units in Escherichia coli via the LacR/O , the TetR/O and AraC/I1-I2 regulatory elements," Nucleic Acids Research, no. 6, pp. 1203-1210, 1997.

[21] H. Backes, C. Berens, V. Helbl, S. Walter, F. Schmid, and W. Hillen, "Combinations of the alpha-helix-turn-alpha-helix motif of TetR with respective residues from LacI or 434Cro: DNA recognition, inducer binding, and urea- dependent denaturation," Biochemistry, no. 18, pp. 5311-5322, 1997.

[22] L. Granger, E. O'Hara, R. Wang, F. Meffen, K. Armstrong, S. Yancey, P. Babitzke, and S. R. Kushner, "The Escherichia coli mrsC gene is required for cell growth and mRNA decay." Journal of Bacteriology, no. 7, pp. 1920-1928, 1998.

[23] O. Scholz, P. Schubert, M. Kintrup, and W. Hillen, "Tet repressor induction without Mg2+," Biochemistry, no. 35, pp. 10914-10920, 2000.

[24] T. Lederer, M. Takahashi, and W. Hillen, "Thermodynamic analysis of tetracycline-mediated induction of Tet repressor by a quantitative methylation protection assay." Analytical Biochemistry, no. 2, pp. 190-6, 1995

[25] P. Schubert, K. Pfleiderer, and W. Hillen, "Tet repressor residues indirectly recognizing anhydrotetracycline," European Journal of Biochemistry, no. 11, pp. 2144-2152, 2004.

[26] W. Hillen, C. Gatz, L. Altschmied, K. Schollmeier, and I. Meier, "Control of expression of the Tn10-encoded tetracycline resistance genes. Equilibrium and kinetic investigation of the regulatory reactions," Journal of Molecular Biology, no. 3, pp. 707-721, 1983.

[27] U. Alon, An introduction to systems biology: design principles of biological circuits. Boca Raton, Florida: Chapman \& Hall, 2006.

[28] W. Hillen, G. Klock, I. Kaffenberger, L. Wray, and W. Reznikoff, "Purification of the TET repressor and TET operator from the transposon Tn10 and characterization of their interaction." Journal of Biological Chemistry, no. 11, pp. 6605-6613, 1982.

[29] O. T. Chis, J. R. Banga, and E. Balsa-Canto, "Structural identifiability of systems biology models: A critical comparison of methods," PLoS ONE, no. 11, 2011. 